\title{
Micro Three-dimensional Removal Processing inside Sapphire substrate
}

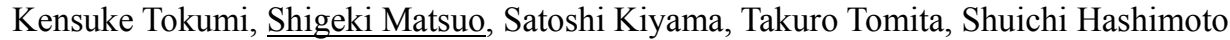 \\ Department of Ecosystem Engineering, The University of Tokushima, Tokushima 770-8506, Japan \\ E-mail: matsuos@eco.tokushima-u.ac.jp
}

Femtosecond laser-assisted etching is a promising technique as micro three-dimensional removal processing. This technique consists of two-steps: the first step is irradiation of focused femtosecond (fs) laser pulses along the pre-designed pattern, and next step is wet etching. Provided the modified region is etched faster than the un-modified host material, the modified region is selectively removed. However, when this technique was applied to volume etching of sapphire, there was a problem of incomplete removal (residues remain after etching). In the present report, we propose and demonstrate a new strategy of two-cycle process, i.e., repeating \{irradiation-etching cycle two-times. The region that should be removed was divided into two. Outer layer was etched at the first cycle and inner volume was etched at the second cycle. In this way, the etching capability was improved as well as suppressing undesirable side effects of cracks and surface pits.

DOI:10.2961/jlmn.2010.02.0015

Keywords: Femtosecond laser processing, Femtosecond laser-assisted etching, sapphire, two-cycle process

\section{Introduction}

One of the unique capabilities of femtosecond (fs) laser processing is three-dimensional (3D) processing in the micro to submicrometer domain. Many researches on 3D microfabrication with photopolymers have been reported using multi-photon absorption [1,2].

Processing inside transparent solid materials have also been researched. Irradiation of single or multiple fs laser pulses changes the refractive index as well as chemi$\mathrm{cal} /$ structural property of transparent solids at small vicinity of the focus. Hereinafter, such a modified spot is referred to as voxel. Typical size of a voxel is about a few micrometers parallel to the optical axis and a few hundred

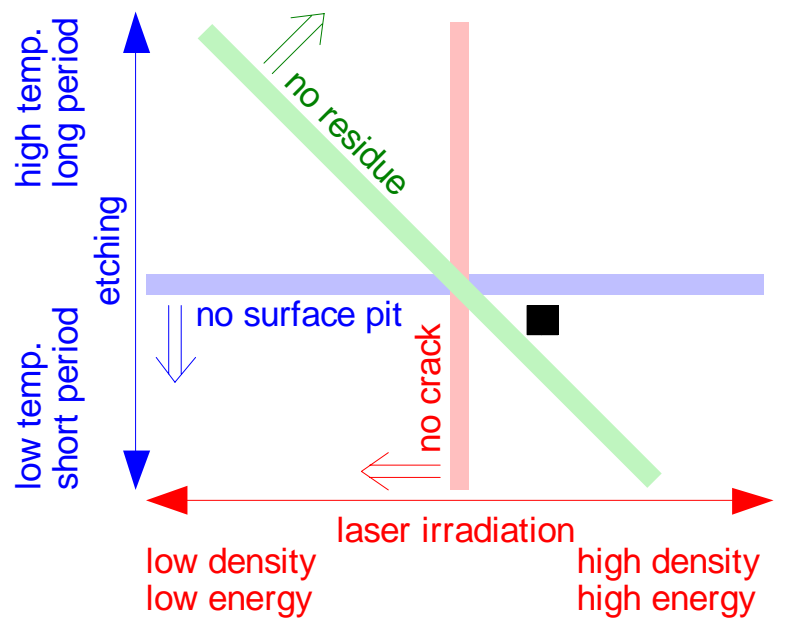

Figure 1 Schematic diagram of property of sapphire for fs laser-assisted etching [11]. Thick lines are borderlines of three issues (see Sec. 1); corresponding double arrows show the desirable region. The black square indicates the condition used in the two-cycle process (see Sec. 2). nanometers perpendicular to the optical axis. By arranging voxels in pre-designed arbitrary 3D pattern, we can make optical elements inside solids such as waveguides [3] and diffractive lenses [4].

Removal processing has been also carried out by making voxels followed by chemical etching. 3D removal processing has been reported for both photosensitive [5] and non-photosensitive [6] materials. This technique has a unique capability of removal process for arbitrary 3D pattern in micrometer domain. Its achievements include a micro dye laser [7] and a ship-in-a-bottle optical rotator.[8].

Sapphire is an important material due to its mechanical strength, chemical durability, optical transparency, etc. Its application field includes optical window, substrate for growth of semiconductors. We have applied fs laserassisted etching to sapphire [9-11] using aqueous solution of hydrofluoric acid (HF) as etchant. Microchannels were fabricated [9]. Juodkazis et al. have demonstrated that more soft etchant, aqueous solution of $\mathrm{KOH}$, was also applicable to the formation of microchannels [12]. Gottmann et al. have reported that sub-wavelength ripples that coherently continue from surface to the inside can be found by similar irradiation-etching technique [13]. However, there was difficulty in volume etching; mesh-like residue remained after etching at room temperature [10]. Here, "volume" indicates a region which consists of multiple voxels in all three dimensions. Thus we have applied hightemperature etching. Increasing the etching temperature from room temperature to $\geq 100{ }^{\circ} \mathrm{C}$ enhanced volume etching capability, but problems still remained [11]. For complete removal of volume region, long and high temperature etching is desirable, which results in the formation of pits on the surface. High-density (small pitch; pitch indicates the distance between the neighboring voxels) and high pulse energy irradiation is also effective for complete removal, but it leads to crack formation after irradiation due 
to overlapped stress. Such a trade-off relationship is schematically shown in Fig. 1 [11]. The horizontal axis indicates the irradiation condition; left hand side is desirable for avoiding cracks, which was represented by the red leftwards double arrow. The vertical axis indicates the etching condition; the lower side is desirable for avoiding surface pits, which was represented by the blue downwards double arrow. Conversely, right-upper side is desirable for complete etching, which was represented by the green upperrightwards double arrow. The region that satisfies all three issues may or may not exist around the center, depending on the shape and size of the region that should be etched out.

In this paper, we propose a new strategy of two-cycle process to overcome these problems.

\section{Concept of two-cycle process}

The basic concept of two-cycle process is to repeat (irradiation-etching s cycle two times. At the first cycle, only the bottom (bottom indicates far from the surface) and side walls, both have the thickness of single voxel size, are irradiated and etched. Next, at the second cycle, the inner volume is irradiated and etched. This scheme is illustrated in Fig. 2. In both cycles, the irradiation and etching conditions lie at the black square in Fig. 1, i.e., irradiation with a hard condition and etching with a soft condition. Irradiation with a hard condition helps complete removal with a soft condition, thus avoiding the formation of surface pits. The hard irradiation condition, however, tends to make cracks. We expect that the formation of cracks can be avoided due to i) small thickness of irradiation region in the first cycle, and ii) the presence of empty space outside in the second cycle.

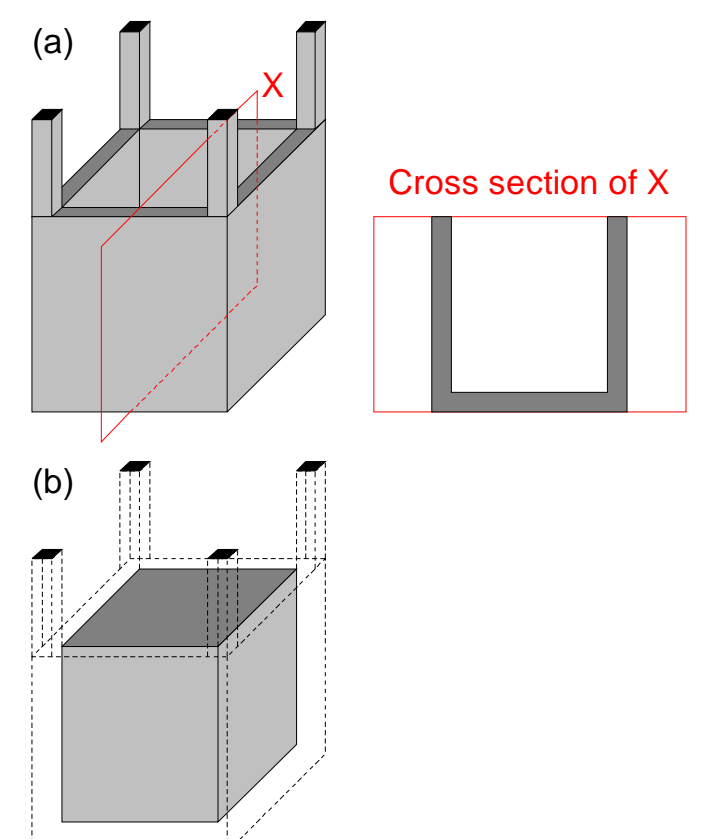

Figure 2 Schematic view of the irradiated region (represented by gray) in the two-cycle process: (a) first cycle, (b) second cycle. Four thin vertical regions are connection paths from the surface to the cubic region that should be removed. The black region on the top of the paths corresponds to the surface of the substrate.

\section{Experimental}

The sample used was sapphire (0001) substrate (Shinkosha Co., Ltd.) of $0.5 \mathrm{~mm}$ thickness. They were cut about $3 \times 3 \mathrm{~mm}$ pieces, and used for experiments.

The fs laser source was a Ti:Sapphire regenerative amplifier (Spitfire, Spectra-Physics) operating with a low repetition rate of $10 \mathrm{~Hz}$ (typ.) for precise positioning. The sample was set on an inverted microscope (IX-70; Olympus) and fs laser pulses $(800 \mathrm{~nm})$ were focused by an objective lens (UPlan-Apo100; Olympus) and irradiated to the sample. The pulse energy was typically 40-60 nJ (measured at the entrance of the optical microscope). The sample was translated by a computer-controlled three-axis piezoelectric stage (P-563.3CD, Physik Instrumente), and laser irradiation was controlled by a shutter.

The region of fabrication target that should be removed out was mainly a cubic region with a edge length of $20 \mu \mathrm{m}$, while the top of the cube lies $10 \mu \mathrm{m}$ below the surface. Hereafter, this cubic region is referred to as microcavity even if the region is not yet empty space. Four connection paths from the surface to the microcavity was also irradiated and etched so that the etchant can reach the microcavity. The spatial distance between adjacent voxels along the optical axis was $1.0 \mu \mathrm{m}$ and that perpendicular to the optical axis was $0.2 \mu \mathrm{m}$ unless specified.

In case of two-cycle process, the outmost voxels on the bottom and side walls were irradiated at the first cycle, and then exposed to the etching process. Then at the second cycle, the inner volume was irradiated. For comparison, irradiation only on the side walls (not bottom wall) at the first cycle was also carried out. For both first and second cycles, etching was carried out at $100{ }^{\circ} \mathrm{C}$ for $24 \mathrm{~h}$ in $10 \%$ aqueous solution of HF. In the former experiments, we did not find surface pits after etching for $96 \mathrm{~h}$ at $100{ }^{\circ} \mathrm{C}$. Thus the formation surface pits can be avoided with this condition.

The sample was observed by optical microscopy in each step, and by scanning electron microscopy (SEM) at the final stage. The SEM observation was carried out for the precise observation inside microcavity. Before the SEM observation, the top solid layer was removed by mechanical polishing.

\section{Results and Discussion}

Figure 3 shows the optical micrographs of the microcavity during two-cycle process. Figure 3(a) shows the optical micrograph after laser irradiation of the first cycle, (b) after etching of the first cycle, (c) after laser irradiation of the second cycle, and (d) after etching of the second cycle. The pulse energy for the first cycle was $40 \mathrm{~nJ}$. Although there are cracks outside of the corners shown in (a), the crack is reduced compared to that of the conventional single cycle process, i.e., all the region inside the microcavity was irradiated [11]. The growth of crack was not observed in the following processes as shown in (b)-(d) [14]. It has been reported that irradiation of single focused fs pulse with sufficient pulse energy can make microvoid in glass [15]. Such a microvoid is also formed in sapphire [16]. Densified region should be formed around the microvoid. Then, it is presumed that the outside of the densified region was under tensile stress in the circumferential direc- 
tion. Even if single microvoid does not cause the formation of cracks, accumulation of tensile stress from many voxels could results in the formation of cracks especially at the corners. The result shown in Fig. 3 indicates that not only outmost voxels but also inner voxels contribute to the crack formation. However, crack did not completely disappear. Further improvement is necessary in the process parameters.

After etching of the first cycle, the inner volume of the microcavity was irradiated by focused fs pulses of $60 \mathrm{~nJ}$ pulse energy, and etched again. Figure 4 shows SEM images after two-cycle process. As stated previously, the surface layer was mechanically polished and inside of the microcavity was directly observed by SEM. As seen in Fig. 4(a1) and (a2), the cubic microcavity region was well etched out; no residue was found. This indicates that the two-cycle process worked well. The crack, found after the first process, is not so conspicuous in the SEM image.

Bottom and side walls of the microcavity, however, are quite rough. This is disadvantageous for optical and fluidic applications. For photosensitive glass, Cheng et al. have shown that the etched surface can be smoothed by thermal annealing after etching [17]. Also it is well known that sapphire substrates can be flattened to the atomic scale by thermal annealing [18]. Although the surface obtained in the present experiments was rough, such thermal annealing might reduce the surface roughness.

We have also tested that irradiation only side walls (without bottom wall) at the first cycle. In this case, increase in the crack was found after laser irradiation of the second cycle. This indicates mechanical separation with the bottom wall is required for the reduction of the propagation of stress from inner volume to the outside.

Figure 4(b) shows a SEM image of microcavity, in which a rectangular column exists. Although residue remained on the bottom, this result indicates the applicability of the present method to the fabrication of arbitrary shape.
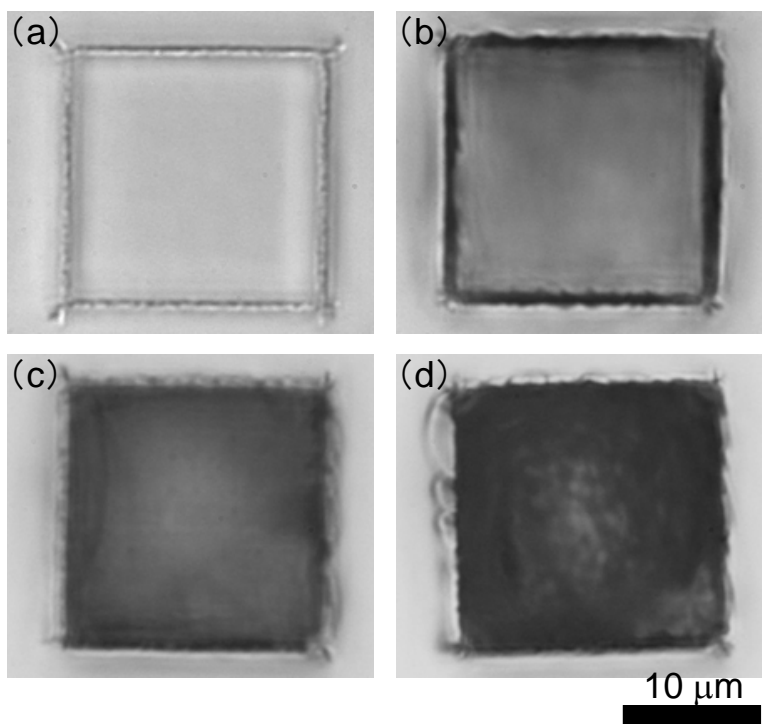

Figure 3 Optical micrographs of microcavity. (a) after laser irradiation of the first cycle, (b) after etching of the first cycle, (b) after laser irradiation of the second cycle, (b) after etching of the second cycle. Scale is common for all.
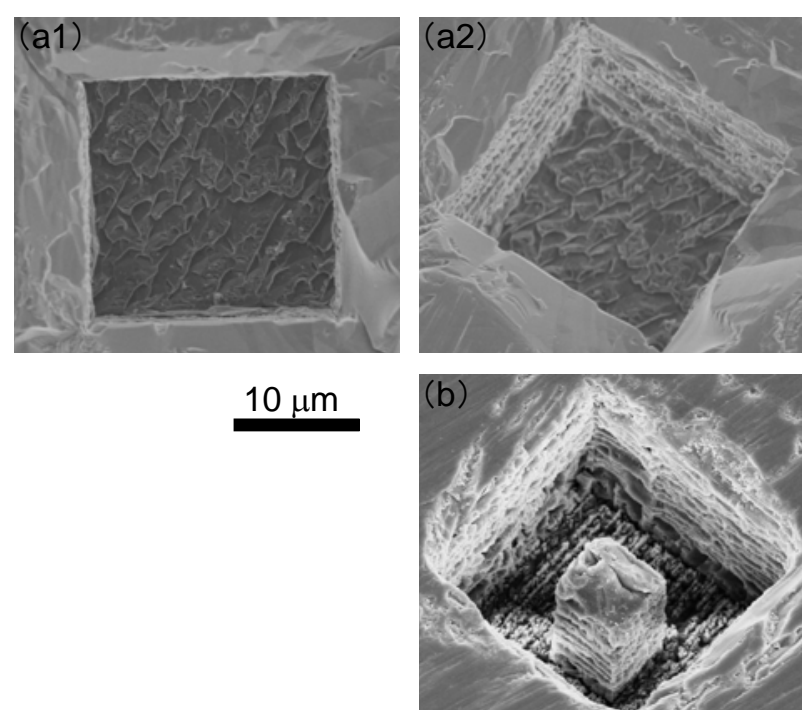

Figure 4 SEM images of (a1) top and (a2) oblique views of a microcavity inside sapphire prepared by the two-cycle process. (b) SEM image of microcavity with a rectangular column. Scale is common for all.

\section{Conclusion}

We have proposed and demonstrated two-cycle femtosecond laser-assisted etching for the processing of sapphire. Using two-cycle process, the volume etching inside sapphire was achieved while formation of surface pits was avoided and crack generation was reduced This technique will be effective for the micro removal processing of arbitrary 3D design inside sapphire.

\section{Acknowledgments}

This work was partly supported by KAKENHI (20360115), Nippon Sheet Glass Foundation for Materials Science and Engineering, and the Venture Business Laboratory of the University of Tokushima.

\section{References}

[1] S. Juodkazis, V. Mizeikis, and H. Misawa: Adv. Polymer Science, 213, (2008) 157.

[2] H.-B. Sun and S. Kawata: Adv. Polymer Science, 170, (2004) 169.

[3] K. M. Davis, K. Miura, N. Sugimoto, and K. Hirao: Opt. Lett., 21, (1996) 1729.

[4] W. Watanabe, D. Kuroda, K. Itoh, and J. Nishii: Opt. Express, 10, (2002) 978

[5] Y. Kondo, J. Qiu, T. Mitsuyu, K. Hirao, and T. Yoko: Jpn. J. Appl. Phys. 38, (1999) L1146.

[6] A. Marcinkevičius, S. Juodkazis, M. Watanabe, M. Miwa, S. Matsuo, H. Misawa, and J. Nishii: Opt. Lett., 26, (2001) 277.

[7] Y. Cheng, K. Sugioka, and K. Midorikawa: Opt. Lett., 29, (2004) 2007.

[8] S. Matsuo, S. Kiyama, Y. Shichijo, T. Tomita, S. Hashimoto, Y. Hosokawa, and H. Masuhara: Appl. Phys. Lett., 93, (2008) 051107.

[9] S. Juodkazis, K. Nishimura, H. Misawa, T. Ebisui, R. Waki, S. Matsuo, and T. Okada: Adv. Mater., 18, (2006) 1361.

[10] S. Matsuo, Y. Shichijo, T. Tomita, and S. Hashimoto: J. Laser Micro/Nanoengineering, 2, (2007) 114. 
[11] S. Matsuo, K. Tokumi, T. Tomita, S. Hashimoto: Laser Chem., 2008, (2008) 892721.

[12] S. Juodkazis, Y. Nishi, and H: Misawa: physica status solidi (RRL), 2, (2008) 275.

[13] J. Gottmann, D. Wortmann, and M. HöstmannJungemann: Appl. Surf. Sci., 255, (2009) 5641.

[14] The asymmetrical formation in cracks observed in Fig. 3(d) (cracks are observed mainly in left side of of microcavity) may be related to the asymmetry in fs laser pulse. See: P.G. Kazansky, W. Yang, E. Bricchi, J. Bovatsek, A. Arai, Y. Shimotsuma, K. Miura, and K. Hirao: Appl. Phys. Lett. 90, (2007) 151120 and W. Yang, P.G. Kazansky, Y. Shimotsuma, M. Sakakura, K. Miura, and K. Hirao: Appl. Phys. Lett. 93, (2008) 171109.
[15] E. N. Glezer, M. Milosavljevic, L. Huang, R.J. Finlay, T.-H. Her, J.P. Callan, and E. Mazur: Opt. Lett., 21, (1996) 2023.

[16] S. Juodkazis, K. Nishimura, S. Tanaka, H. Misawa, E.G. Gamaly, B. Luther-Davies, L. Hallo, P. Nicolai, and V.T. Tikhonchuk: Phys. Rev. Lett., 96, (2006) 166101.

[17] Y. Cheng, K. Sugioka, K. Midorikawa, M. Masuda, K. Toyoda, M. Kawachi, and K. Shihoyama: Opt. Lett., 28, (2003), 1144.

[18] M. Yoshimoto, T. Maeda, T. Ohnishi, H. Koinuma, O. Ishiyama, M. Shinohara, M. Kubo, R. Miura, and A. Miyamoto: Appl. Phys. Lett., 67, (1995) 2615.

(Received: February 12, 2010, Accepted: May 27, 2010) 